\title{
VIIRS/J1 Polarization Narrative
}

\author{
Eugene Waluschka ${ }^{1}$, Joel McCorkel ${ }^{1}$, Jeff McIntire ${ }^{2}$, David Moyer ${ }^{3}$, Brendan McAndrew ${ }^{1}$, Steven \\ W. Brown ${ }^{4}$, Keith Lykke ${ }^{4}$, James B. Young ${ }^{5}$, Eric Fest ${ }^{6}$, James Butler ${ }^{1}$, Tung R. Wang ${ }^{7}$, Eslim O. \\ Monroy $^{7}$, Kevin Turpie ${ }^{8}$, Gerhard Meister ${ }^{1}$ and Kurtis J.Thome ${ }^{1}$ \\ ${ }^{1}$ NASA/Goddard Space Flight Center, Greenbelt, MD \\ ${ }^{2}$ Science Systems and Applications, Inc., Greenbelt, MD \\ ${ }^{3}$ The Aerospace Corporation, El Segundo, CA \\ ${ }^{4}$ NIST, Gaithersburg, MD \\ ${ }^{5}$ Stellar Solutions, Palo Alto, CA \\ ${ }^{6}$ Raytheon, Tucson, AZ \\ ${ }^{7}$ Raytheon, El Segundo, CA \\ ${ }^{8}$ University of Maryland, Baltimore County
}

\begin{abstract}
The VIS/NIR bands polarization sensitivity of Joint Polar Satellite Sensor 1 (JPSS1) Visible/Infrared Imaging Radiometer Suite (VIIRS) instrument was measured using a broadband source. While polarization sensitivity for bands M5-M7, I1, and I2 was less than 2.5\%, the maximum polarization sensitivity for bands M1, M2, M3, and M4 was measured to be $6.4 \%, 4.4 \%, 3.1 \%$, and $4.3 \%$, respectively with a polarization characterization uncertainty of less than $0.3 \%$. A detailed polarization model indicated that the large polarization sensitivity observed in the M1 to M4 bands was mainly due to the large polarization sensitivity introduced at the leading and trailing edges of the newly manufactured VISNIR bandpass focal plane filters installed in front of the VISNIR detectors. This was confirmed by polarization measurements of bands M1 and M4 bands using monochromatic light. Discussed are the activities leading up to and including the instruments two polarization tests, some discussion of the polarization model and the model results, the role of the focal plane filters, the polarization testing of the Aft-Optics-Assembly, the testing of the polarizers at Goddard and NIST and the use of NIST's T-SIRCUS for polarization testing and associated analyses and results.
\end{abstract}

Keywords: JPSS, VIIRS, T-SIRCUS, polarization

\section{INTRODUCTION}

The VISNIR bands polarization sensitivity of Joint Polar Satellite Sensor (JPSS) Visible/Infrared Imaging Radiometer Suite (VIIRS/J1) ${ }^{1}$ instrument was measured using a broadband source. While polarization sensitivity for bands M5-M7, $\mathrm{I} 1$, and I2 was less than $2.5 \%$, (the requirement is bands M1, I2, M7 less than $3 \%$ and bands M2, M3, M4, I1, M5, M6 less than 2.5\%) the polarization sensitivity for bands M1, M2, M3, and M4 was measured to be $6.4 \%, 4.4 \%, 3.1 \%$, and $4.3 \%$, respectively with a polarization characterization uncertainty less than $0.3 \%$. The expectation was that VIIRS/J1 would, as did VIIRS/NPP, meet its polarization requirements. The unexpectedly large polarization sensitivity resulted in the following activities:

- determine the root cause of the increased polarization sensitivity using a detailed polarization ray trace model (using the commercial code FRED);

- to determine by means of the polarization ray trace model that the increased polarization sensitivity was due to the focal plane filters leading and trailing spectral bandpass edges (which differed from VIIRS/NPP);

- find ways to ascertain the correctness of the models predictions and the need to get a better understanding on the uncertainty of the measurements;

- repeat a subset of the original polarization measurements including new telescope scan angles to estimate "scan angle" interpolation errors;

- $\quad$ erform a series of polarization measurements using monochromatic light provided by NIST's tunable lasers (T-SIRCUS) to verify the model's predictions. 
The following sections describe briefly, in somewhat chronological order the activities associated with the polarization measurements, the results and the analysis of the observed polarization sensitivity of VIIRS/J1.

For historical reasons, the VIIRS program uses some non-standard terminology when referring to the polarization sensitivity of the system. The translation between VIIRS and standard Mueller matrix terminology ${ }^{2}$ is given in Table A1.

\section{VISNIR OPTICAL PATH}

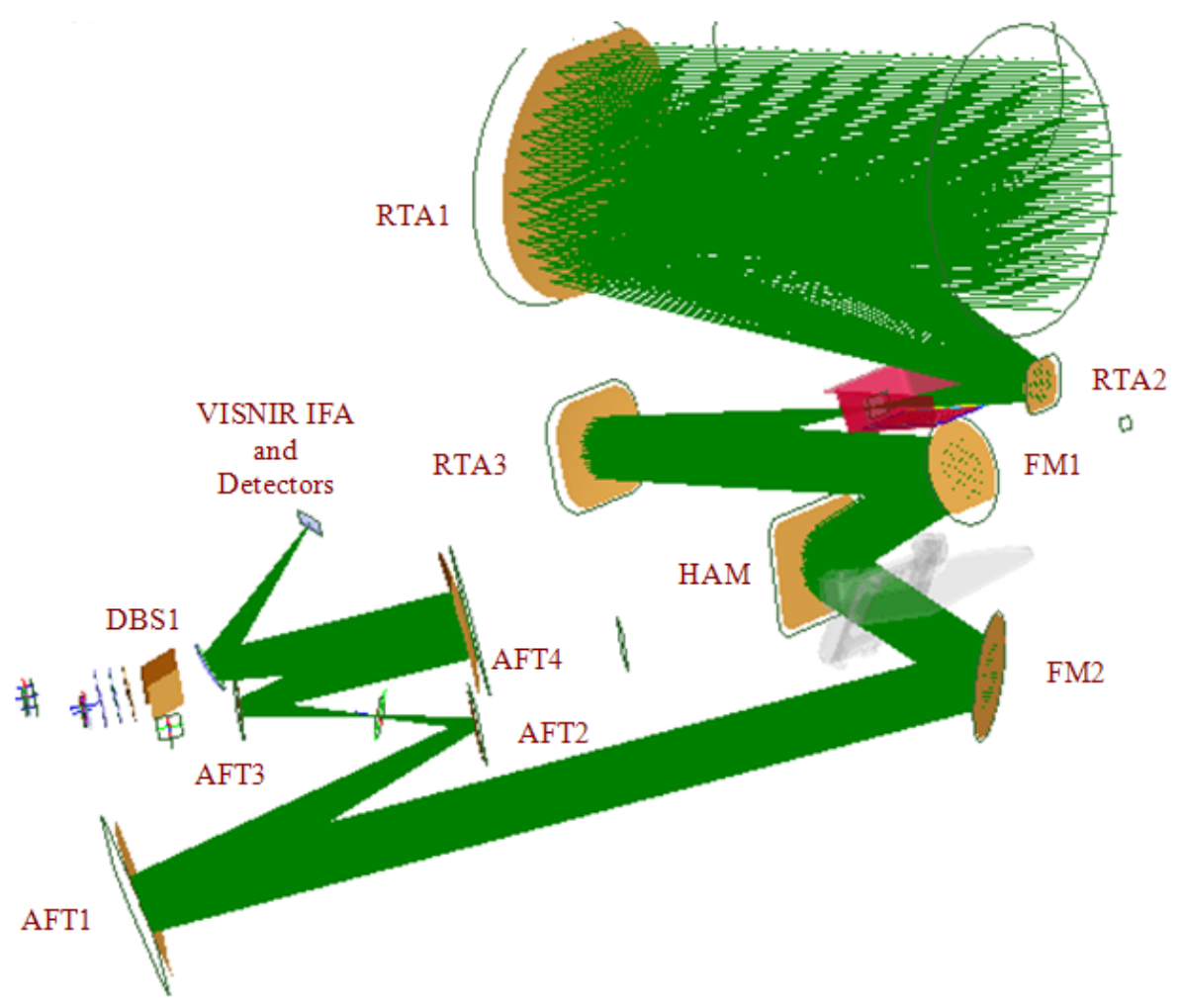

Fig. 1. VISNIR optics within the VIIRS/J1 FRED model

To orient the reader to subsequent sections we start with the VIIRS VISNIR optics, shown in fig. 1, which shows all of the optics in the plane of the paper, but in reality they are not. The rotating telescope (a cross track scanner) consists of three powered elements and a flat fold mirror which directs the light onto the rotating "half-angle" mirror (HAM), which is a two sided rotating fold mirror. The half angle mirror, rotating at half the speed of the telescope de-rotates the light beam and directs it onto the aft-optics. It should be noted that the HAM is the only optic with a varying - during scanning - angle of incidence; hence it is the only optic which can change the polarization sensitivity during scanning! The aft-optics assembly (AOA) consists of the four powered elements, AFT1 to AFT4 followed by the dichroic \#1 (DBS1) which reflects the visible and near-IR light toward the VISNIR/DNB (Day Night Band) focal plane filter assembly which is immediately above the detectors. The image on the focal plane is about the size of a $35 \mathrm{~mm}$ slide.

\section{AFT-OPTICS-ASSEMBLY (AOA) POLARIZATIOIN TEST}

The polarization sensitivity of the VIIRS/J1 AOA was measured in fourth quarter of $2012^{3}$. This is motivated by the fact that VIIRS/NPP instrument level polarization ${ }^{4}$ measurements performed in 2009 shown that, even though the instrument met its polarization requirement, there was non-negligible detector-to-detector $(\mathrm{d} 2 \mathrm{~d})$ variation in polarization sensitivities in bands M1 and M4.Subsequent test confirmed the $\mathrm{d} 2 \mathrm{~d}$ variation was real and the test approach and equipment was not the source of the observed $\mathrm{d} 2 \mathrm{~d}$ variation. This $\mathrm{d} 2 \mathrm{~d}$ variation, if it is real, would require a separate 
atmospheric correction for each detector, not just for a single band dependent correction for the entire array. Polarization ray trace modeling of VIIRS/NPP was performed but the results were inconclusive. By performing the polarization sensitivity measurement on AOA where fewer optical elements were involved than the whole sensor would help to identify which optical element(s) could cause a d2d variation in the VIIRS/J1 instrument.

The AOA polarization sensitivity measurement was performed with following arrangement: the AOA was looking into a spherical integrating sphere with a polarizer in-between, i.e.:

$$
\text { Spherical Integrating Source }=>\text { polarizer }(\text { theta })=>\text { AOA }
$$

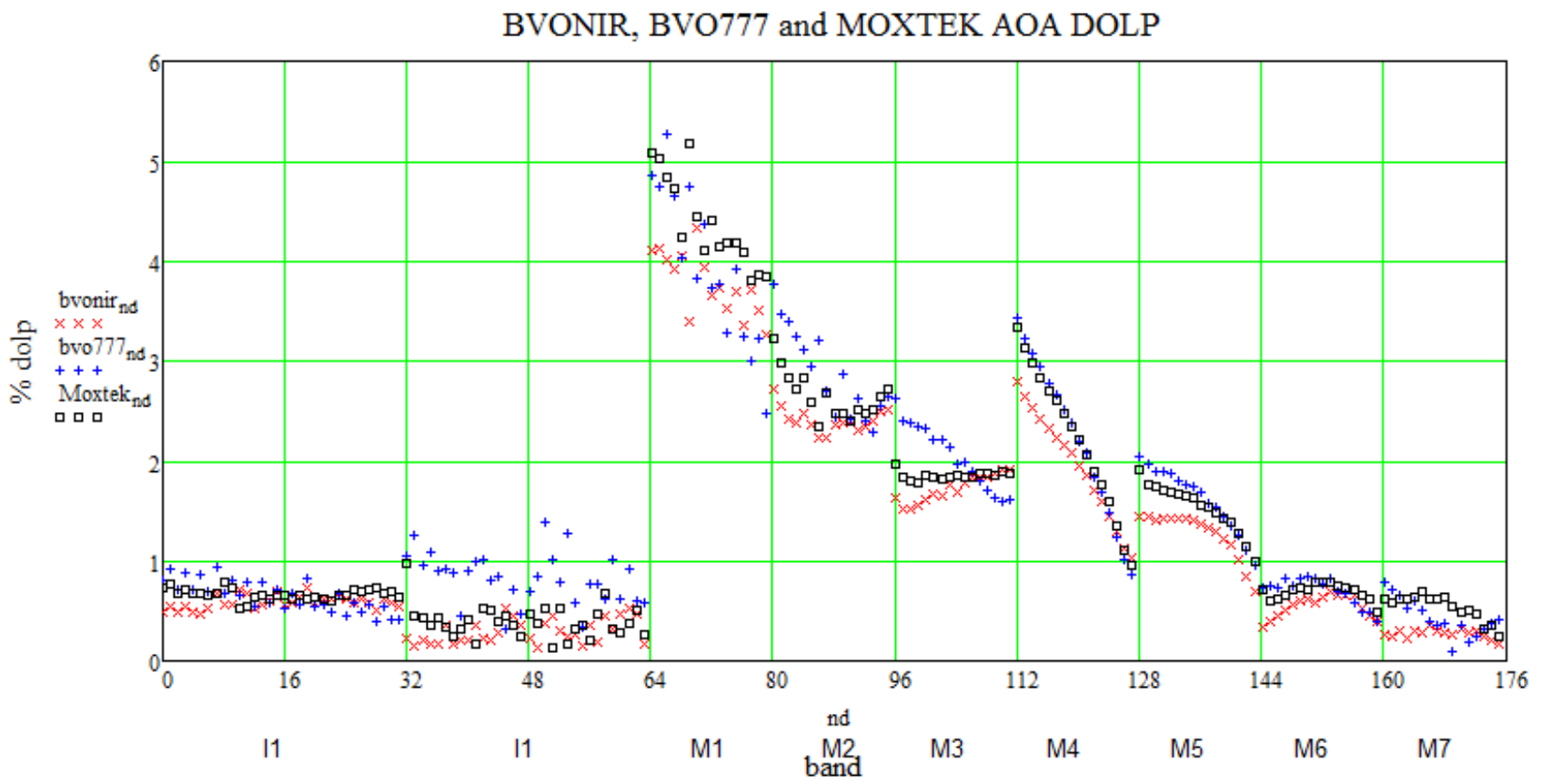

Fig. 2. Band and detector Dolp

Three polarizers were used: Bolder Vision Optik's ${ }^{5}$ BVO777 and BVONIR sheet polarizers, and a sufficient size Moxtek $^{6}$ wire grid polarizer to cover the AOA entrance aperture.. The polarizers were rotated from 0-360 degrees in 15 degree steps. From the resulting two theta intensity variation, the sensitivity of the AOA to linearly polarized light, "DoLP" was determined. Each polarizer measurement was performed twice, By-and-large the measurements repeated well considering the magnitude of the random noise which increased when the two theta sinusoidal variations were small.. The BVONIR and BVO777 measurements were corrected for polarization efficiency determined by cross polarization measurement of two polarizers of the same type, one fixed and one rotating, where AOA is used as a detector. (For some of the BVO777 measurements a Sonoma ${ }^{7}$ long wave blocking filter was used to reduce the possible IR leakage for M1 to M3 bands) The Moxtek efficiency was not measured since its' polarization efficiency was deemed high. . As shown in fig.2, the results using the Moxtek wire polarizer, even without polarization efficiency correction, were in line with the corrected sheet polarizer measurements

The three sets of data plotted in fig. 2 show results of the measurements using the BVONIR, the BVO777 sheet and the Moxtek wire grid polarizer. Each point in the plot is an averaged detector response from two repeated measurements. All three polarizers give reasonably consistent result for all of the bands. The I1 and I 2 bands ( 32 detectors) exhibited the most noise because the two theta variation was small. Band M1 and M4 show a pronounced $\mathrm{d} 2 \mathrm{~d}$ variation. Band M1, even though it has the most polarization sensitivity, between 3 and 5 percent, was inexplicably noisy.

Due to the concern that BOVNIR polarization sensitivity measurement might be contaminated by stray light contribution, these AOA results was not viewed as representative of the system level polarization sensitivity. 


\section{REASON FOR POLARIZATION REQUIREMENT}

The instrument response is required to vary less than $2.5 \%$ when viewing fully polarized light being measured for all reflective solar bands, except I2, M1 and M7, for which the requirement criterion is set at $3 \%$ Raytheon $(2008)^{8}$. The degree of polarization from molecular scattering for VISNIR light observed above the atmosphere can be as high as 60 to $70 \%$, depending on the wavelength. The resulting variation in instrument response can thus be greater than $1.5 \%$ (or $2 \%$ in the case of band M1). This creates geometrically depended artifacts in the satellite imagery for science data products that are sensitive to radiometric errors. In particular, retrieval of band-specific, water-column reflectance below the air-water interface, or ocean color remote sensing reflectance, is sensitive enough to require a correction, based on laboratory characterization of the instrument polarization response, to the Top-of-Atmosphere (TOA) radiance or reflectance measurements.

This correction is essential to calculating accurate ocean color remote sensing reflectance (or water-leaving radiance), from which all traditional ocean color data products are derived. Without this correction, the ocean data products would show strong scan dependent biases and striping that would vary seasonally and hemispherically across the globe. Previous experience with MODIS Aqua showed that this could also impact global and synoptic trends, thus adversely affecting these products as climate data records.

Both NOAA and NASA ocean color processing systems have the capability of apply this correction to the ocean products (NOAA EDRs or NASA Level-2 products, respectively) through the correction factor $p_{c}$, defined as

$$
p_{c}=\frac{s_{m}}{s_{t}}
$$

where $L_{m}$ and $L_{t}$ are defined as the measured and true TOA radiance, respectively. For most scan angles, $p_{c}$ can be calculated as

$$
P_{g}=\frac{1}{1-m_{\mathrm{ca}} Q_{\mathrm{g}} / L_{\mathrm{m}}-m_{\mathrm{cs}} E_{\mathrm{t}} / L_{\mathrm{m}}}
$$

where $Q_{t}$ and $U_{t}$ are the modeled Stokes Vector components of the TOA radiance associated with molecular scatter. For scan angles close to nadir, a matrix rotation is needed, as described by eq. 5 in Meister et al. $(2005)^{9}$. This method has been employed for ocean color for a long time and experience with it is extensive. Gordon et al. $(1997)^{10}$ originally developed this correction approach, and its implementation is practically identical for both MODIS and VIIRS (except, of course, for the values of the coefficients $m_{12}$ and $m_{13}$, which are derived from the prelaunch characterization of the respective instruments). The polarization response correction is applied to the TOA radiance during NOAA EDR or NASA Level-2 processing because of the availability of Rayleigh scatter model being used in the atmospheric correction.

\section{WORK AT NIST AND GODDARD CHARACTERIZING POLARIZERS}

The BVO777 and BVONIR sheet polarizers were used to measure the VIIRS/NPP/J1 polarization sensitivity. The large area of the sheet polarizers enables the required full aperture polarization testing. From polarizer efficiency considerations, for the VIIRS/NPP/J1 measurements, the BVO777 was preferred over the BVONIR polarizer for the shorter wavelengths, although both produced similar results over all of the VIS/NIR wavelength during instrument level tests and in the preliminary tests, at Raytheon, measuring the polarization of a tilted glass plate, albeit with different error bars. However, BVO777 has a small polarization efficiency at M7/I2 bands and BVONIR is preferred at these wavelengths..

The appearance of the BVO777 sheet is clear and it does not put any great requirements on the shape or alignment of the circular exit aperture of the Spherical Integrating Source (SIS). Whereas, the BVONIR is white and diffuse in appearance and it does require that the SIS aperture be circular (because of the asymmetric scattering of light) and aligned with the optical axes of the SIS and VIIRS. The AOA polarization test used a smaller, with a circular aperture, sphere, than that used for the instrument level tests. 
Several polarizing elements were characterized at GSFC in order to verify that the expected polarization signal can be retrieved using a monochromatic source. The recent (last couple of years) VIIRS-related polarizer efficiency measurements were performed at Goddard and NIST. The typical measurement setup is:

SIS $=>$ limiting aperture $=>$ polarizer-1(fixed) $=>$ polarizer-2(theta) $=>$ spectral filter $=>$ detector $=>$ sinusoidal variation

The SIS's light source has been incandescent bulbs or a fiber connected to a monochromatic light source such as the SIRCUS laser. The simplest measurement has both polarizers 1 and 2 of the same type (i.e. BVONIR and BVONIR or Moxtek and Moxtek) as this approach does not require knowledge of the SIS radiance as long as it remains stable or accurately monitored. Another measurement approach is to have polarizer-1 be one with a very high extinction ratio. Either approach gives the sinusoidal signal from which the correction factor or extinction ratio is determined. The results of the tests were consistent with the VIIRS/NPP polarizer characterization, and therefore providing confidence that the BVONIR could be used to characterize polarization sensitivity of VIIRS/J1 to required levels of accuracy using a monochromatic source.

For those who have followed the MODIS polarization testing and remember the observed "4 theta" sinusoidal variation $^{11}$, the cause of which was the light, do to telecentricity, reflected from the focal plane back through all of the MODIS optics, into the Polarization Source Assembly reflecting from the back of the Ahrens prism (hence polarized again) and then back into MODIS and producing the not so small "4 theta" variation. This same 4 theta variation was observed in the Moxtek - Moxtek efficiency tests when the separation was small and caused by the light bouncing between the two polarizers.

\section{POLARIZATION TEST SETUP, SPECIAL TEST EQUIPMENT (STE) RESIDUAL POLARIZATION AND POLARIZATION CHARACTERIZATION UNCERTAINTY}

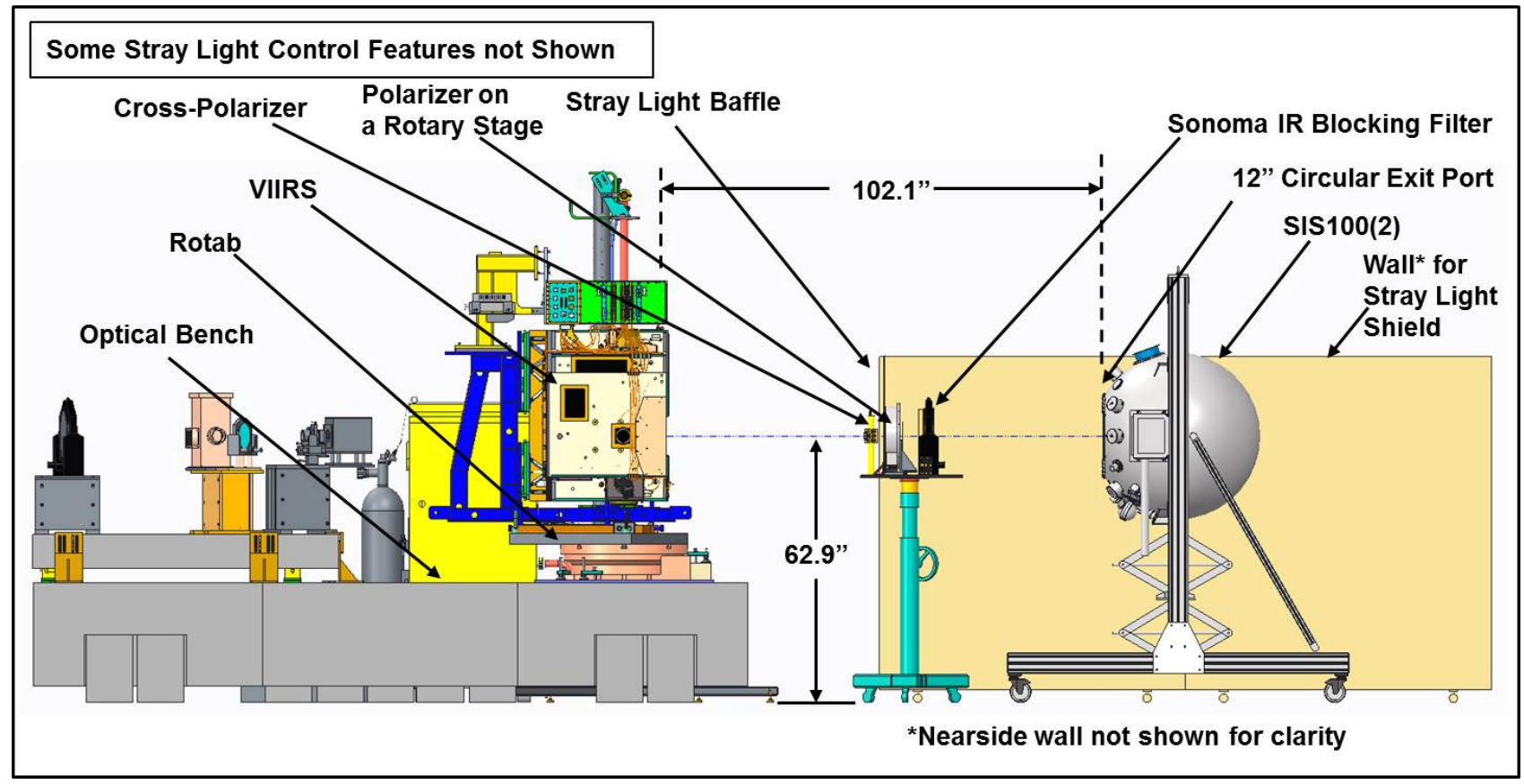

Figure 3. VIIRS Polarization Test Setup

The VIIRS polarization test setup for FP-11, FP-11' and T-SIRCUS is shown in fig.3. The VIIRS sensor was mounted on a Rotab such that it could be rotated in its scan plane and have the scan angle set anywhere in-between $-55^{\circ}$ to $+55^{\circ}$ from nadir. Unpolarized light from the Spherical Integrating Source (SIS) was transmitted through an 11" diameter BVONIR or BVO777 sheet polarizer producing linearly polarized light. The sheet polarizer was mounted to a Newport (RV350PE) rotary stage, tilted $0.6^{\circ}$ toward the gravity, was rotated from $0^{\circ}$ to $360^{\circ}$ (or $0^{\circ}$ to $180^{\circ}$ for the T-SIRCUS test) in $15^{\circ}$ increments. For the shorter wavelength bands (up $488 \mathrm{~nm}$ ), a long-wave blocking filter, Sonoma C165-210-JB, 
was located between the SIS and the polarizer to block wavelengths longer than $\sim 600 \mathrm{~nm}$. The VIIRS line-of-sight (LOS), sheet polarizer, Sonoma filter, and SIS were co-aligned in both positions and angles. A stray light baffle was installed, as shown, in between the SIS and VIIRS, to minimize possible stray light not directly illuminated through the sheet polarizer. Additional baffle walls and Velostat sheets were used to enclose the sheet polarizer and SIS setup to suppress stray light. For the FP-11 and FP-11' tests, a 40" diameter SIS100(2) with a 12" circular exit port was used to generate a white light illumination. For the T-SIRCUS polarization test the SIS100(2) was replaced by a 40" diameter TSIRCUS SIS which also had a 12" circular exit port. The in-situ stray light characterization was performed by collecting data with the SIS on and off and with an obscuration test (A.K.A. the lollipop which entails placing a circular disk, slightly larger than the VIIRS footprint at the polarizer, in front of the sheet polarizer).

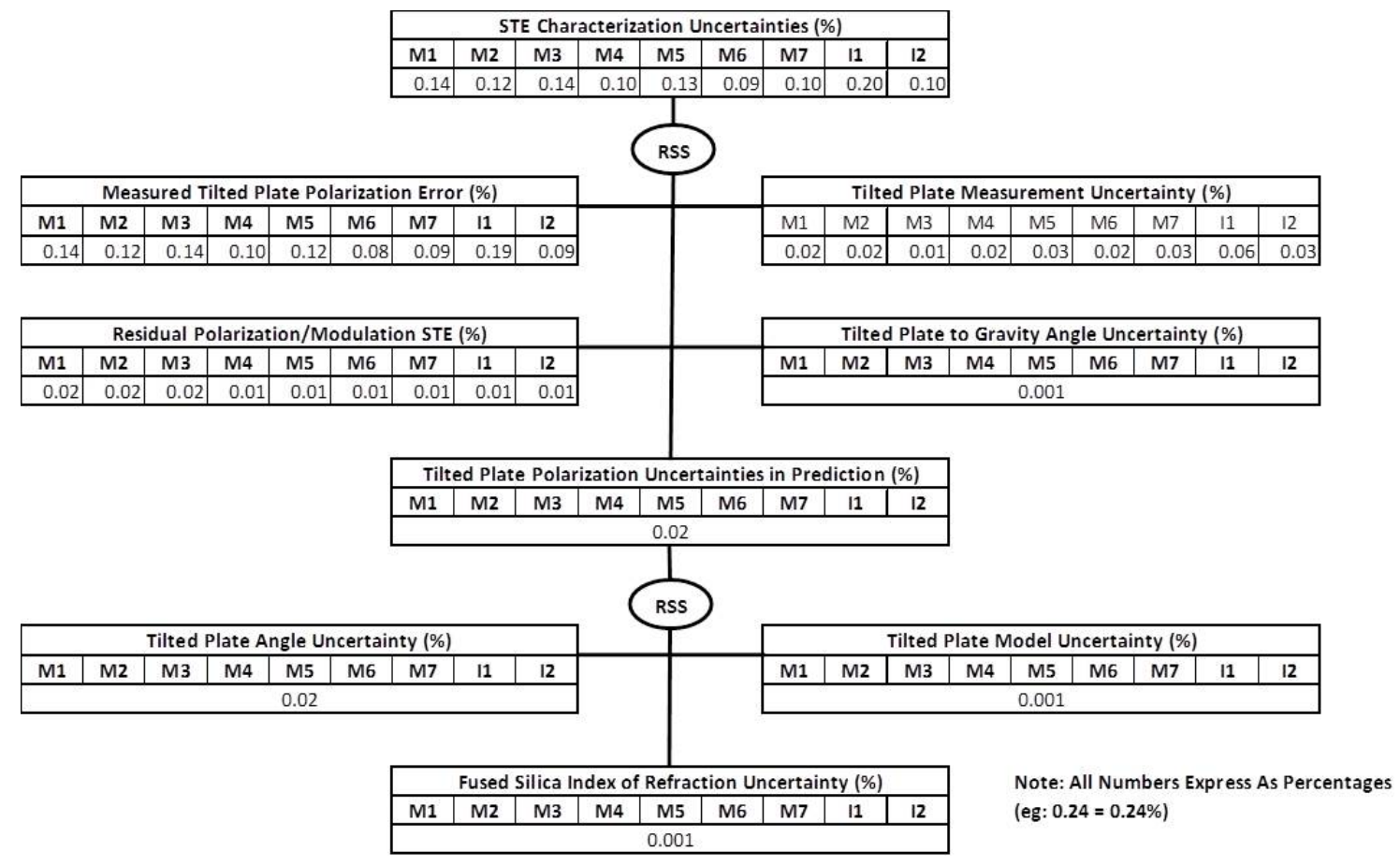

Table 2. STE Residual Polarization

The polarizer sheet efficiency was measured by adding a second, non-rotating sheet polarizer in the optical path and in front of the rotating polarizer, fig.3. The polarizer efficiency test was performed for each sheet polarizer type with both the rotating and non-rotating polarizers being of the same type (i.e. BVO777 with BVO777 and BVONIR with BVONIR).

In the FP-11 and FP-11' tests, for bands M1 to M3 the polarization sensitivity was based on measurements using the BVONIR polarizer plus Sonoma IR blocking filter. For the other VISNIR bands, the results are based only the BVONIR polarizer. For the T-SIRCUS bands M1 and M4 polarization tests, only the BVONIR sheet polarizer was used without Sonoma IR blocking filter since T-SIRCUS generates monochromatic light and there is no need to block the longer wavelengths.

Prior to FP-11 and FP-11' polarization tests, the residual polarization of the testing optics and light source was characterized using the Polarization Test Source Assembly (PTSA) which was mounted on the Rotab in place of VIIRS. The PTSA was designed to have a similar acceptance aperture and effective focal length as VIIRS, with VISNIR band pass filter witness samples placed in front of the PTSA detector to simulate VISNIR band performance.

An additional check on the polarization measurement approach shown in fig. 3 , but with VIIRS replaced by the PTSA (and by similarity to the actual VIIRS measurement), the polarization sensitivity of a 12" diameter fused silica plate, tilted $30^{\circ}$ relative to the VIIRS-polarizer line-of-sight, was measured. The tilted glass plate was placed in-between the 
PTSA and the polarizer and data collected as the polarizer was rotated. Data was also collected with PTSA tilted to $+0.75 \mathrm{mR}$ and $-0.75 \mathrm{mR}$ in the VIIRS track direction to simulate the VIIRS VISNIR detector spatial dependent polarization sensitivity. The measurements and analysis showed that for the VIIRS VISNIR bands the PTSA based polarization measurement uncertainty varied from $0.09 \%$ at M6 band and to $0.2 \%$ at I1 band, Table 2 .

Table 2 shows both estimated $1 \sigma$ rms and worst case (maximum) polarization uncertainties, The worst case uncertainty, a conservative upper bound of $1 \sigma$ uncertainty, for VIIRS instrument measurements varied from $0.13 \%$ on M6 band and to $0.38 \%$ on $\mathrm{M} 1$ band and that this meets the polarization characterization $1 \sigma$ uncertainty requirement of $0.5 \%$ with margin.

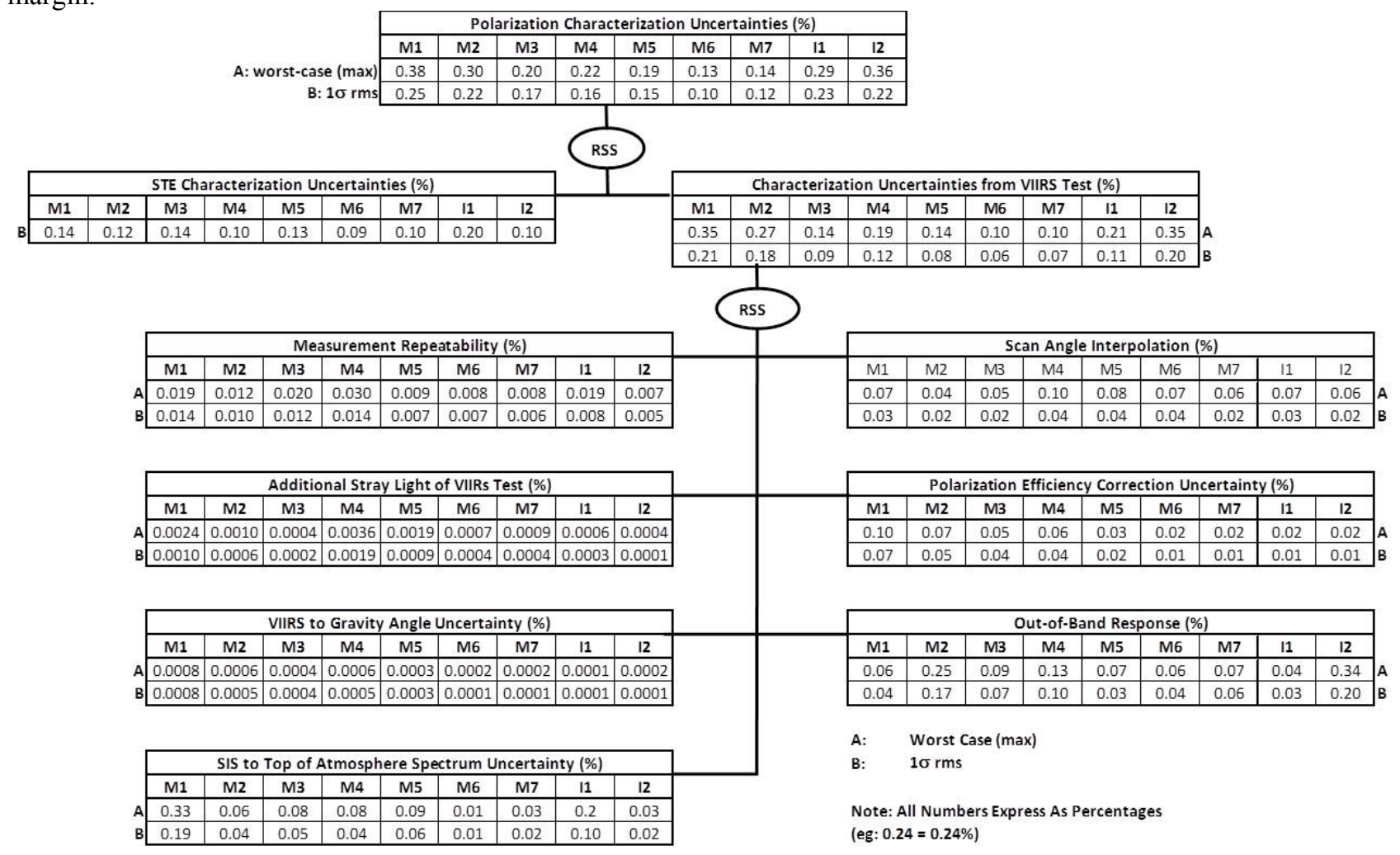

Table 3. VIIRS Polarization Characterization Uncertainty

\section{FIRST POLARIZATION TEST “FP-11”}

The first VIIRS/J1 polarization test, FP-11 $1^{12}$, took place at Raytheon Space and Airborne System (SAS) in El Segundo in $4^{\text {th }}$ quarter of 2013. Test was performed with, the room lights turned off. As shown in fig.3, with VIIRS looking into the SIS100, at scan angles of $-55^{\circ},-45^{\circ},-20^{\circ},-8^{\circ},+22^{\circ},+45^{\circ}$, and $+55^{\circ}$, the polarizer was rotated from $0^{\circ}$ to $360^{\circ}$ in $15^{\circ}$ steps. At each polarizer angle, the detector data in each VISNIR band was recorded. The detector data vs, polarizer angle shows a distinctive " 2 theta" sinusoidal variation. Fitting a 2 theta sinusoid to this data gives the needed Mueller matrix components from which we get, for each detector, the DoLP's which were plotted in fig.4 A comparison of the FP-11 and AOA results, fig. 2 and fig. 4, shows that, even though there is no telescope and HAM in the AOA, the polarization sensitivity results are similar. The AOA data seems noiser and was about $1 / 2$ percent less than the FP-11 results. Fig. 4(a) and 4(b)b show the polarization sensitivity variation with scan angle and the HAM side differences. While band M1 detector 1 shows very little scan angle variation, detector 16 shows about $4 \%$ scan angle variation. As can be seen in Fig. 4, bands M1, M2, M3, and M4 exceed the polarization requirement. 


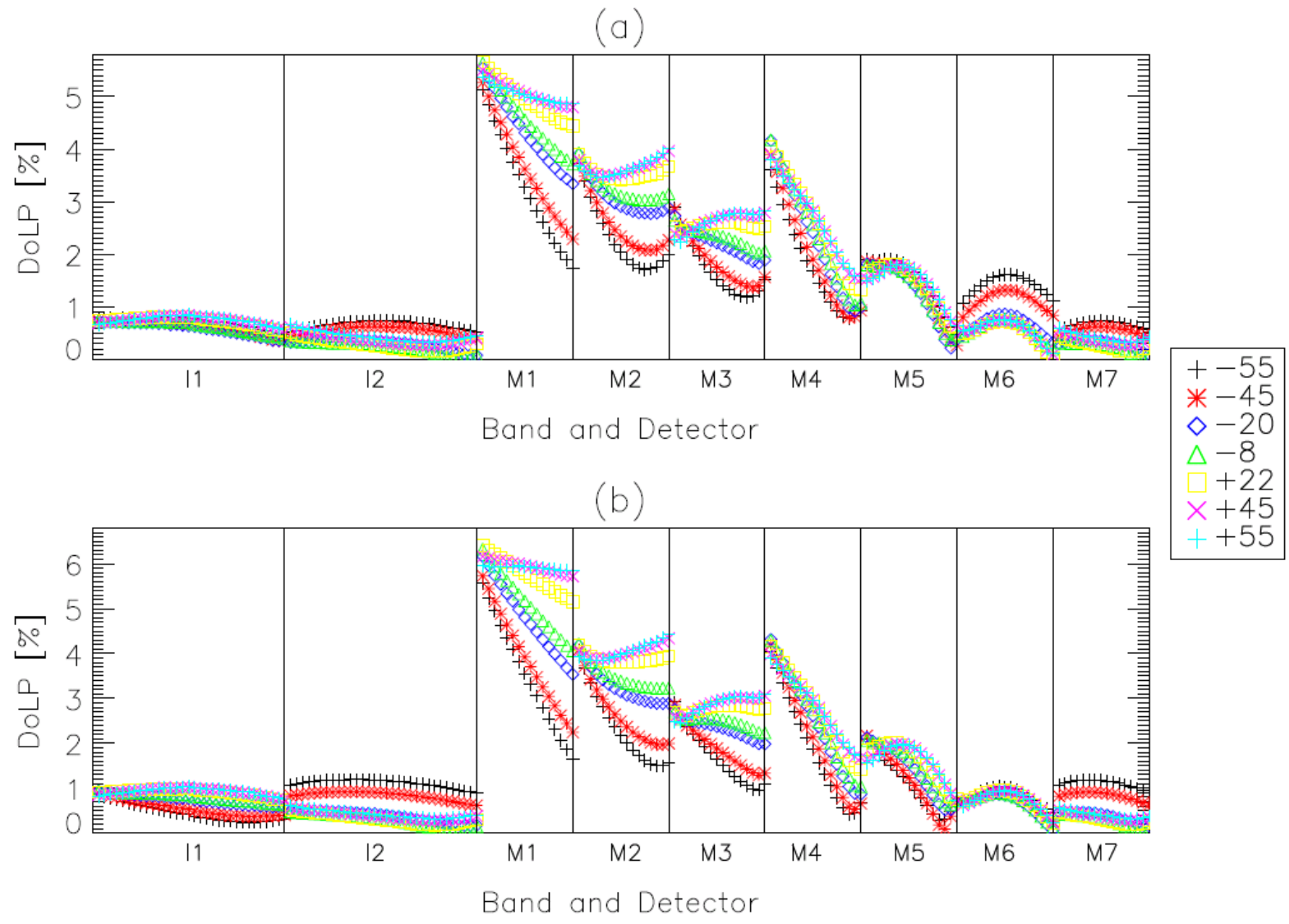

Figure 4: DoLP [\%] results derived from FP-11 testing. The different color / symbol combinations correspond to the different measured scan angles, as shown in the legend. The X-axis shows each band demarked by vertical solid black lines; within each band the DoLP for each detector is plotted in ascending order along the x-axis. Plot (a) shows the results for HAM side 0 and plot (b) graphs the results for HAM side 1.

\section{NOAA/NASA ADDITIONAL POLARIZATION TEST RECOMMENDATION}

J1 VIIRS polarization response test results and NASA-modelled impacts to EDRs were reviewed and discussed by NASA and NOAA scientists. In an effort to fully characterize the impact of the polarization sensitivity and to ultimately enable high-fidelity on-orbit correction, the NASA/NOAA group recommended two additional sets of polarization measurements to be performed in ambient at Raytheon. These measurements included the following:

- polarization sensitivity of bands M1 to M7 at 4 additional VIIRS scan angles using polarized SIS-100 output. For each band, polarization sensitivity was requested to be measured at VIIRS scan angles of $+4,-15,-30$, and 37 degrees. Measurements at -8 degrees were also requested as a scan angle common to previous polarized SIS100 measurements made by Raytheon.

- polarized radiance response as a function of wavelength of bands M1 and M4 using monochromatic polarized T-SIRCUS light. For each band, 13 wavelengths were requested to be measured, with 3 wavelengths near the band center and 5 wavelengths on each side of the band center. These measurements were to be made at 2 VIIRS scan angles for each band. At each wavelength and at the two scan angles, measurements, by VIIRS, of the polarized output of the T-SIRCUS were to be made at 13 polarization angles from 0 to 180 degrees in steps of 15 degrees.

The data from the measurements at the additional scan angles would be used to validate the consistency and smoothness of the polarization sensitivity data previously acquired by Raytheon and would provide confidence in post-launch EDR corrections. The radiance response measurements would be used to quantify the effect of polarization on the shape of VIIRS response curves and validate the predictions of the Raytheon J1-VIIRS polarization response model for those bands. The Raytheon model would be used to determine polarization effects at scan angles not measured in ambient 
conditions at Raytheon, evaluate impacts from polarization changes in band spectral shape, and identify causes of onorbit changes in polarization sensitivity.

\section{FRED MODEL AND ITS PREDICTIONS}

A model of the VIIRS/J1 optical system was created in the FRED optical analysis program and is shown in Fig.1. The optical prescription used in this model is identical to the NPP model ${ }^{13}$.

In the J1 model the mirror coatings are virtually the same as in the NPP mode. fig 5a. shows the difference between the $\mathrm{s}$ and $\mathrm{p}$ reflectance $\left(\mathrm{R}_{\mathrm{s}}-\mathrm{R}_{\mathrm{p}}\right) / 2$ (which is a measure of polarization sensitivity and is equal to the unpolarized transmittance * linear diattenuation) of the J1 mirrors and NPP mirrors. However, the difference between $\mathrm{s}$ and $\mathrm{p}$ transmittance $\left(T_{s}, T_{p}\right) / 2$ of the J1 IFA filters, fig. 5b-i, is significantly higher than the NPP filters, especially at the edges of the spectral bands. This difference is an unintended consequence of the redesign of the filters (which was done to improve manufacturability and equalize the response across the VISNIR bands) and is significant even at an Angle of Incidence (AOI) of 13 degrees, which is the highest angle the in-field ray bundle makes with the IFA. Though the phenomenon of polarization splitting at the edges of bandpass filters has been documented ${ }^{14}$, an assumption was made that this would not be a significant effect in VIIRS because of the low AOI on the IFA, and therefore there was no component-level specification for maximum allowed polarization splitting was defined for it. As shown in Fig.6 this increase in polarization splitting for J1 significantly increases its polarization sensitivity relative to NPP, especially for the M1-M4 bands. The figure shows that the model accurately predicts the J1 polarization sensitivity to within $1.6 \%$ in bandaveraged DoLP To achieve this accuracy it was necessary to sample each waveband with 25 wavelengths across each waveband, as measured from the $5 \%$ unpolarized transmittance points. The predicted polarization sensitivity vs. wavelength is shown in Figure 6; this data is necessary for calibrating the sensor's Relative Spectral Response (RSR) ${ }^{15}$.
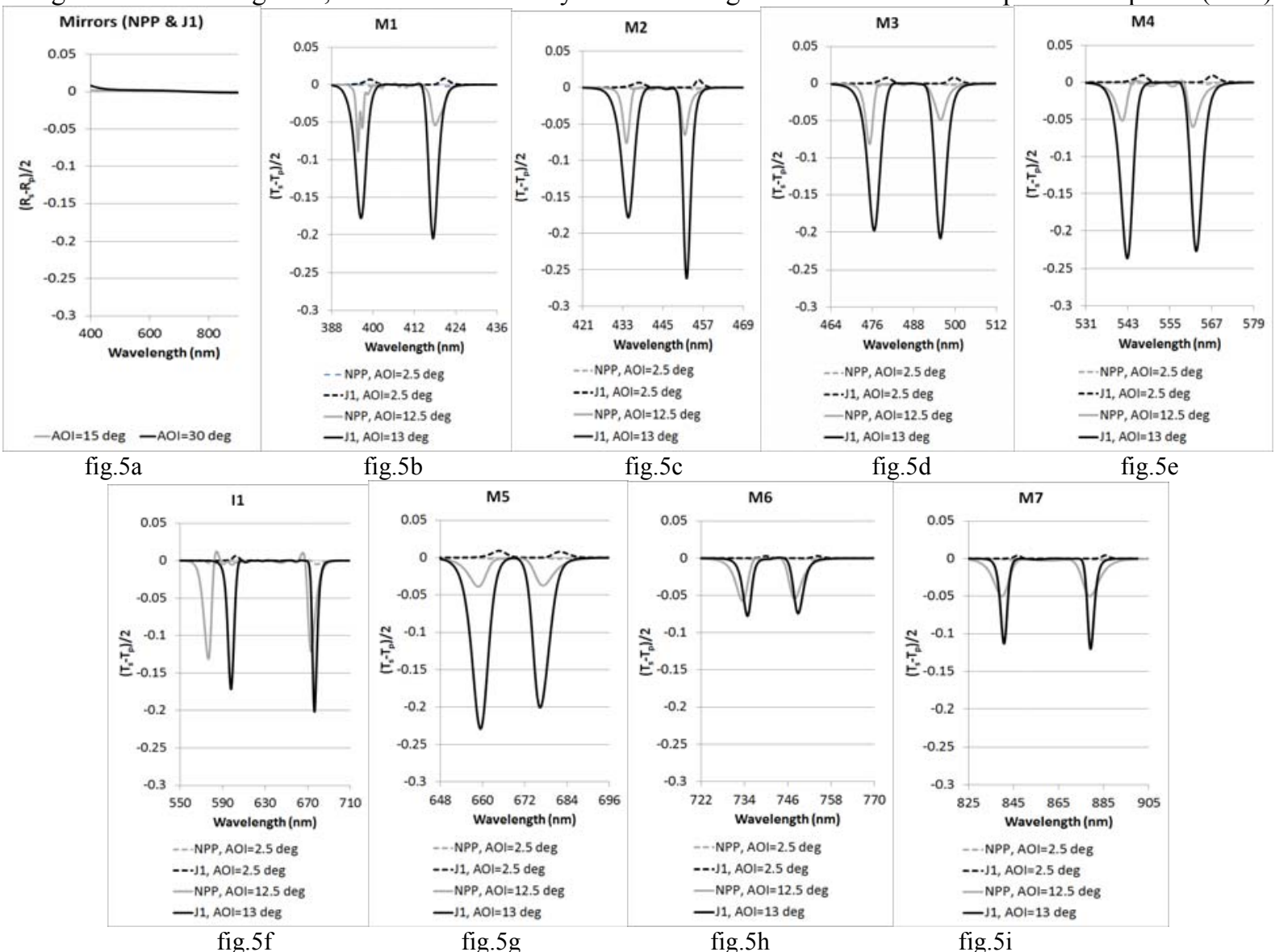

fig. $5 b$

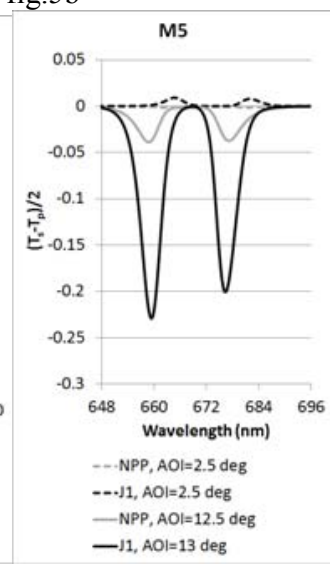

fig. $5 \mathrm{c}$

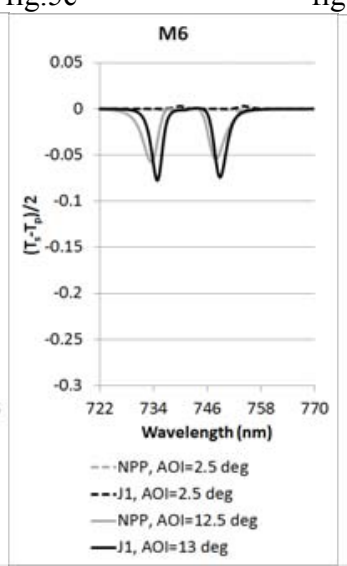

fig. $5 \mathrm{~h}$ fig. $5 \mathrm{~d}$ fig.5e

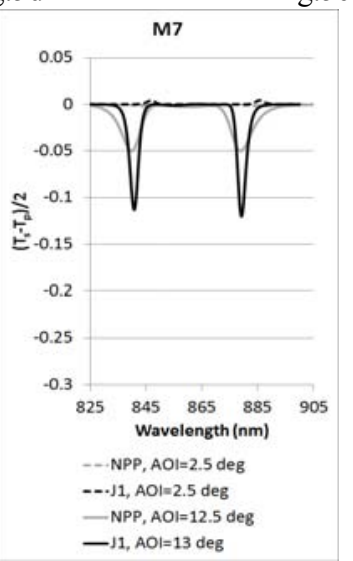

fig.5i

Figure 5. Predicted $\left(\mathrm{R}_{\mathrm{s}}-\mathrm{R}_{\mathrm{p}}\right) / 2$ of the NPP and $\mathrm{J} 1$ mirrors, and $\left(\mathrm{T}_{\mathrm{s}}-\mathrm{T}_{\mathrm{p}}\right) / 2$ of the NPP and J1 IFAs.. 


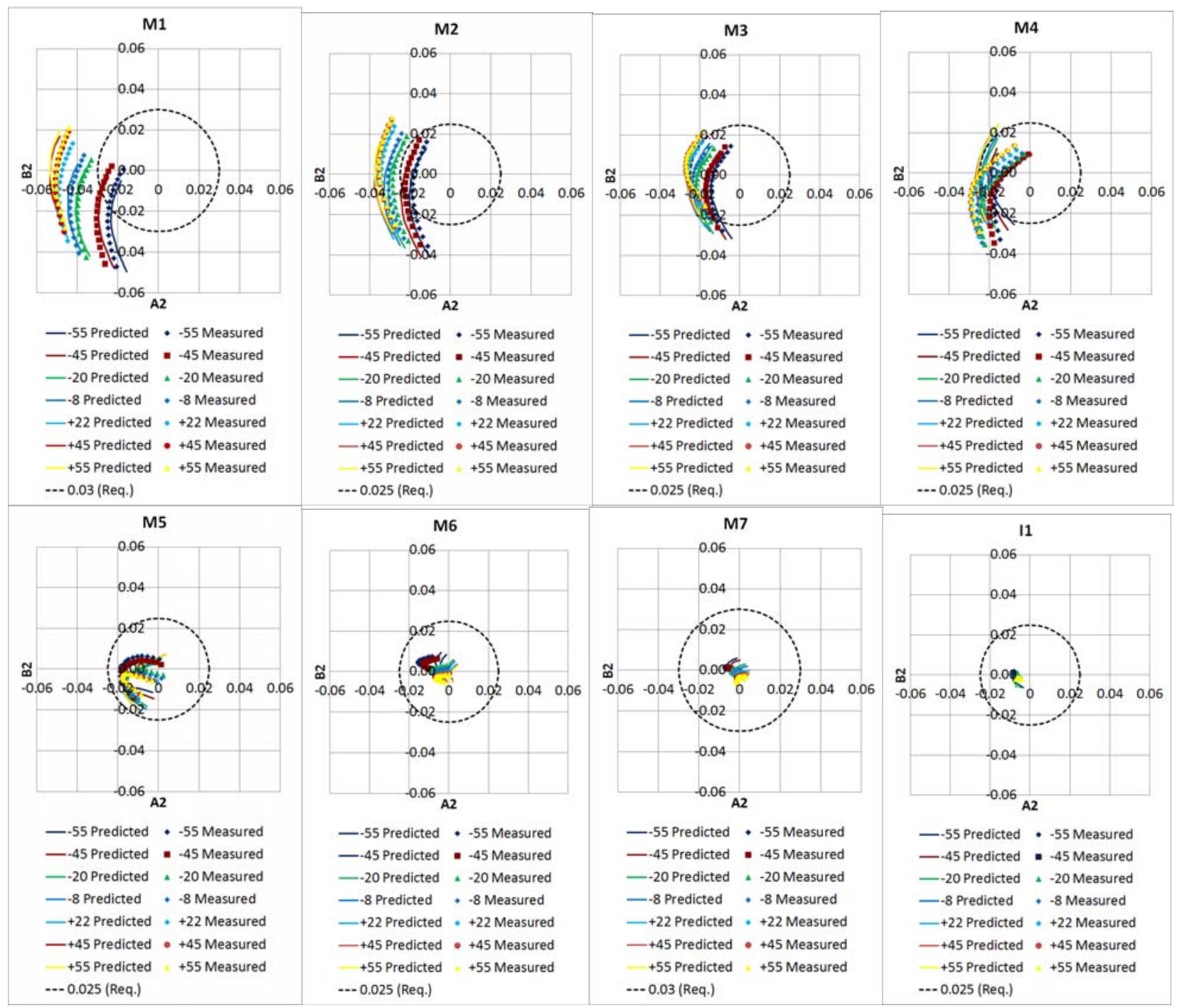

Figure 6. Predicted and Measured HAM-A J1 Polarization Sensitivity (Band-Averaged). The predictions are made using the product of the measured spectra of the Spherical Integrating Source (SIS) and either the Sonoma bandpass filter, and BVO777 polarizer (bands M1-M3 only) or the BVONIR polarizer (M4-M7) used in the FP-11 test.

In the previous analysis performed for NPP [4], only the center wavelength of each waveband was used, and this resulted in an error between the predicted and measured polarization sensitivity. This analysis was rerun using 25 wavelengths per band resulting in much better correlation $(<0.5 \%$ for most bands) between the predicted and measured polarization sensitivity.

\section{ANALYSES AND ACTIVITIES BETWEEN FP-11 AND FP-11'AND T-SIRCUS}

It has been generally accepted that the TOA correction for ocean color data products should work if the sensor polarization response is relatively small and has been characterized to within $0.5 \%$ uncertainty (i.e., within instrument requirements). ${ }^{7}$ However, although J1 VIIRS characterization uncertainty for polarization response has been claimed to be within this uncertainty threshold, the response observed for this sensor exceeded instrument requirements, and in some cases exceeded that of heritage sensors (especially at $551 \mathrm{~nm}$ ). Furthermore, it was apparent that changes in the spectral response across each band pass could further degrade the correction effectiveness. Therefore, a sensitivity analysis of a highly stressing scenario for characterization error was employed to determine whether the assumption that 
the characterization uncertainty was adequate and to evaluate the potential impact of apparent the spectral properties of the J1 VIIRS polarization response. ${ }^{16}$

\section{SECOND POLARIZATION TEST FP-11'}

The second VIIRS polarization test, FP-11', took place in Raytheon SAS in El Segundo in $3^{\text {rd }}$ quarter of 2013. The test configuration was the same as in FP-11. The polarization sensitivity test results, including the repeated measurement at $8^{\circ}$ scan angle, are shown Fig.7., - The polarization sensitivity measurement at $-8^{\circ}$ scan between FP-11 and FP-11' was very similar. The small differences at $-8^{\circ}$ scan angle between FP-11 and FP-11' results is consistent with estimated polarization measurement accuracy.

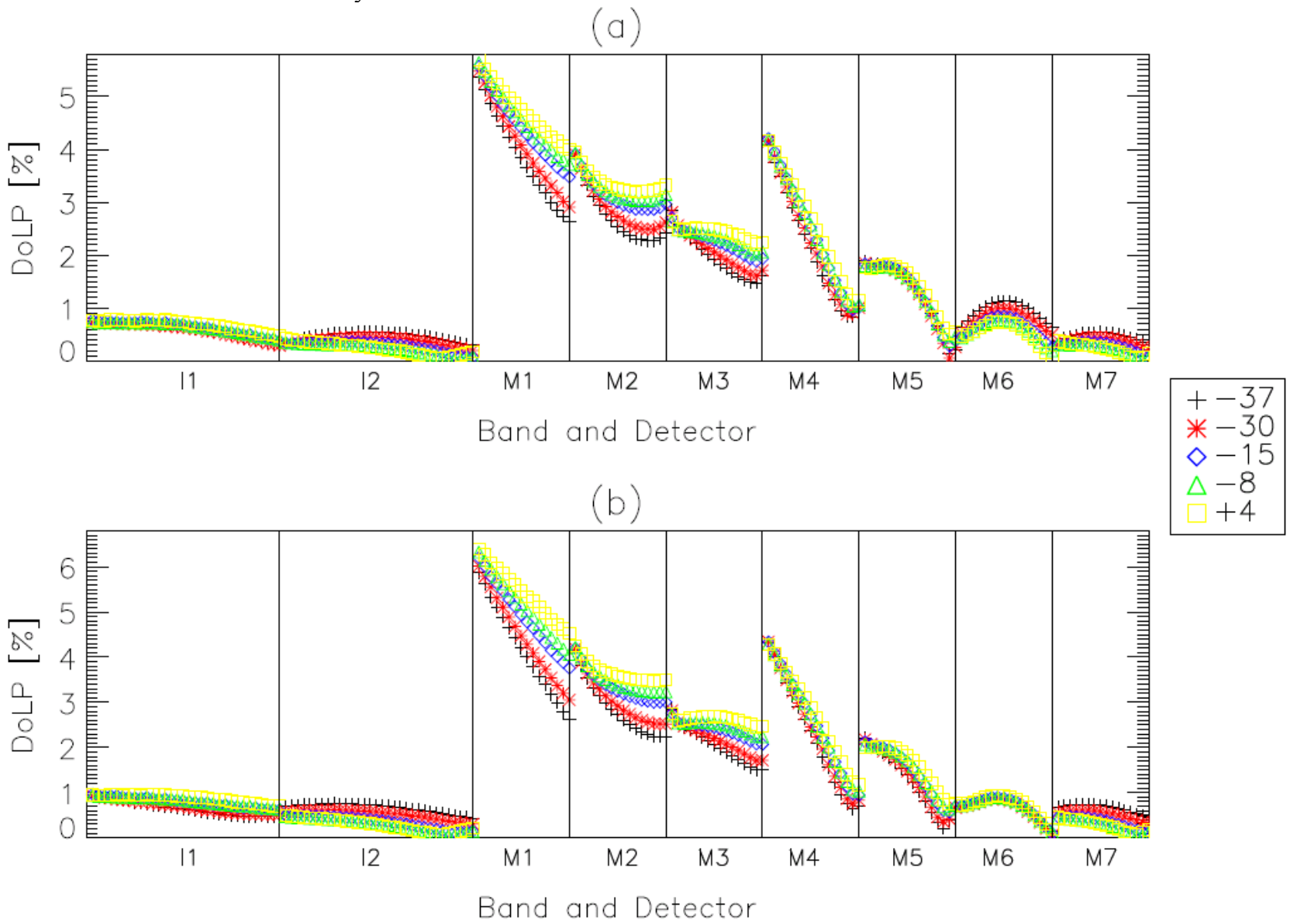

Figure 7: DoLP [\%] results derived from FP-11' testing. The different color / symbol combinations correspond to the different measured scan angles, as shown in the legend. The $\mathrm{x}$-axis shows each band demarked by vertical solid black lines; within each band the DoLP for each detector is plotted in ascending order along the x-axis. Plot (a) shows the results for HAM side 0 and plot (b) graphs the results for HAM side 1 .

\section{VIIRS SCAN ANGLE INTERPOLATION}

VIIRS on-orbit data analysis requires knowledge of the polarization sensitivity at all scan angles. However the ground characterization (FP-11) measured the polarization at only the following scan angles:

$$
-54.83^{\circ},-44.92^{\circ},-19.85^{\circ},-7.94^{\circ},-7.94^{\circ},-7.94^{\circ}, 21.95^{\circ}, 45.06^{\circ}, 54.99^{\circ} \text {. }
$$

(The repeated angles indicate measurements made at the same scan angle as checks on measurement repeatability.) Interpolation is required for other angles. The interpolation is accomplished by fitting a second order polynomial to the 
two theta Fourier coefficients A2 and B2 (corrected by A0) for each detector. These coefficients are used to calculate the DoLP and phase for each spectral band, detector, at all scan angles.
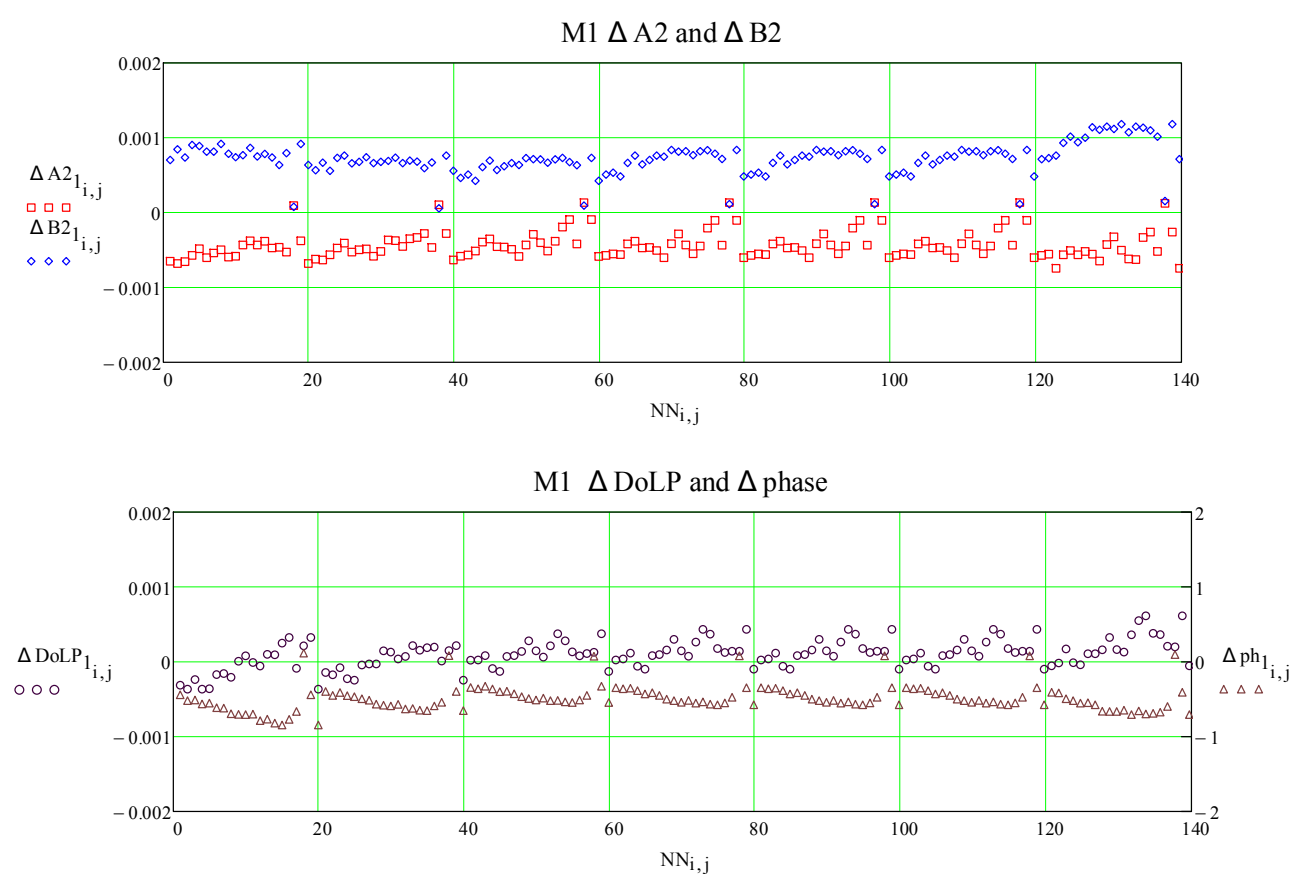

Fig. 8; Differences between FP-11 and FP-11' A2 and B2 (Mueller matrix elements) and Dolp

It was found that the second order polynomial fit to the measured A2 and B2 over the all scan angles had residuals of less than 0.001. Since this fit is a critical parameter, it was decided that an additional test was needed to validate the FP11 results. The second instrument polarization measurement, FP-11', used the same FP-11 polarization test equipment, but the measurements were performed at the following different scan angles:

$$
-36.99^{\circ},-29.98^{\circ},-15.02^{\circ},-7.98^{\circ},-7.98^{\circ},-7.98^{\circ}, 4.00^{\circ} \text {. }
$$

The FP-11 A2 and B2 polynomials were used to calculate the DoLP and phase at the FP-11' scan angles. In addition, FP-11' A2' and B2' polynomials were used to calculate the DoLP, and phase at measured FP-11' scan angles. The differences between the two sets of A2, B2, DoLP and phase were calculated and plotted in fig. 8 shows the results for spectral band M1. The major tick marks on the abscissa delineate the seven FP-11 scan angles and the points between the tick marks is the data for the $16 \mathrm{M} 1$ detectors.

Use of FP-11 A2 and B2 polynomials has shown that VIIRS polarization performance can be predicted at intermediate scan angles: A2 values are within +/- 0.001 and B2 values are within +/- 0.001. In general, the DoLP is within +/-0.0005 except for M4 which is within +0.0005 to -0.0013 and for band M5 the variation is within 0 to -0.001 .

\section{T-SIRCUS SET UP AND OPERATION}

The T-SIRCUS system, the traveling version of the Spectral Irradiance and Radiance Calibration using Uniform Sources facility ${ }^{17,18}$, was developed by NIST to radiometrically calibrate instruments at higher spectral brightness than possible with prior lamp and monochromator based techniques. It consists of an integrating sphere for generating uniform illumination, high accuracy transfer radiometers, fiber coupled tunable laser based light sources, and control and monitoring electronics. 

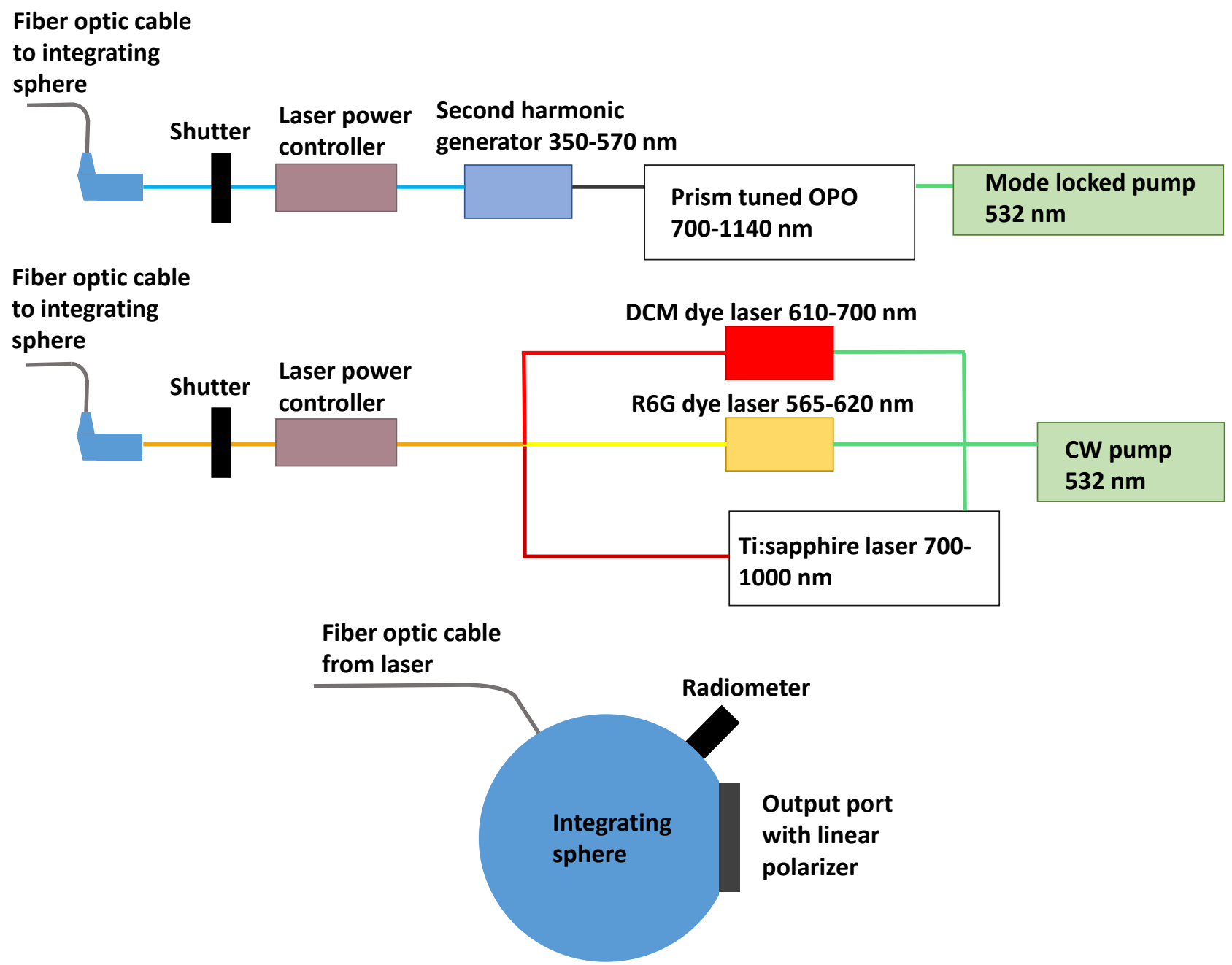

Figure 9: T-SIRCUS set up

For the VIIRS polarization sensitivity tests using the T-SIRCUS, a 40" diameter SIS with a 12" circular exit port was used to generate a monochromatic light illumination which overfill VIIRS's aperture while a transfer radiometer mounted directly to the sphere serves as a radiance monitor and provides feedback for a laser power stabilizer. Using the sphere monitor as a feedback source rather than sampling a portion of the free space beam improves stability as it compensates for variations in fiber coupling due to vibration. The correspondence between radiance at the output port of the sphere and monitor position was checked both before and after instrument calibration by comparing response between the sphere monitor and a second transfer radiometer mounted in front of the sphere. The radiometers are of the silicon trap detector type and are temperature stabilized during measurements. They were calibrated over the wavelength range of interest using absolute radiometric facilities at NIST.

Several lasers and optical parametric oscillators were used to tune across the spectrum. The short end of the wavelength range from $350-570 \mathrm{~nm}$ was covered with the second harmonic of a custom optical parametric oscillator developed at NIST and pumped at $532 \mathrm{~nm}$ by a 20 watt frequency doubled $\mathrm{Nd}: \mathrm{VO}_{4}$ laser. The pump laser is mode-locked, providing pulses of several picoseconds duration at $80 \mathrm{MHz}$ repetition rate. The oscillator output is therefore also pulsed at 80 $\mathrm{MHz}$, but is treated as continuous wave during the calibration as it is well above the instrument frequency response. The integrating sphere also provides some temporal averaging of pulses. There is a small tuning gap near $532 \mathrm{~nm}$ when the oscillator is operating near its degeneracy of $1064 \mathrm{~nm}$, but this is in an out of band region of VIIRS where small tuning steps were not needed. 
The remainder of the visible spectrum is covered with two dye lasers; Rhodamine 6G for $565-620 \mathrm{~nm}$ and DCM for $610-700 \mathrm{~nm}$. Both were pumped by an 18 watt continuous wave frequency doubled $\mathrm{Nd}: \mathrm{VO}_{4}$ laser. This laser also served as the pump source for a titanium sapphire laser which covered the remainder of the spectrum from $700-1000$ nm.

\section{T-SIRCUS (MONOCHROMATIC) SPECTRAL POLARIZATION MEASUREMENT}

The reason for performing monochromatic polarization measurements of VIIRS has its roots in the fact that the VIIRS $\mathrm{J} 1$ optical filters were redesigned from NPP filters to reduce out-of-band effects. The redesigned filters have, as it turns out, significant polarization across the spectral band, in particular at the spectral band edges. (The " $\mathrm{S}$ " and "P" transmission at the spectral band edges differ.) When the Raytheon polarization model, as described above, takes this into account, the model prediction and FP-11 and FP-11' results for band M1 is good, but for band M4 the agreement could be better. To check the correctness of the J1 model's predictions and increase the confidence that the J2 focal plane filters (redesigned with the aid of the polarization model to reduce the band edge polarization) would result in less polarization sensitivity the monochromatic, T-SIRCUS, polarization measurements were performed. Additionally the TSIRCUS polarization measurements would provide information (because we would be getting within band and not simply band averaged polarization sensitivities) about how the TOA and SIS100 spectral distribution affect VIIRS polarization performance.

The T-SIRCUS polarization measurements were, because of time and cost constraints, limited to the measurements of only two bands, $\mathrm{M} 1$ and $\mathrm{M} 4$, and only at the scan angles $-8^{\circ}$ and $+45^{\circ}$. The reason for this choice was that for band M1 model and measurements agreed well and band M4 (important for the ocean color community and accurate correction for instrumental polarization is required) agreement was poorer. Originally 13 monochromatic polarization measurements were to be performed for each band, with five wavelengths on the band edges and 3 on the band plateau. But as M4 testing progressed, 4 additional plateau measurements were added verify the presence of polarization sensitivity not seen in both the component measurements and consequently the FRED model.

With the T-SIRCUS test data in hand, a fourth order Fourier series was used to fit the sensor response over polarization angle. The Fourier components were used to determine the DoLP and phase angle. This was done separately for each detector, wavelength, and scan angle. The Fourier components were weighted by the spectral transmittance of the system, and then integrated over the bandpass to determine the band dependent DoLP and phase angle for comparison to FP-11 / FP-11' polarization measurements. A more extensive discussion of the T-SIRCUS results are in "Analysis of JPSS J1 VIIRS Polarization Sensitivity Using the NIST T-SIRCUS".

$\Delta$ DoLP FP-11 minus T-SIRCUS/SIS spectrum ( sonoma filter- center)

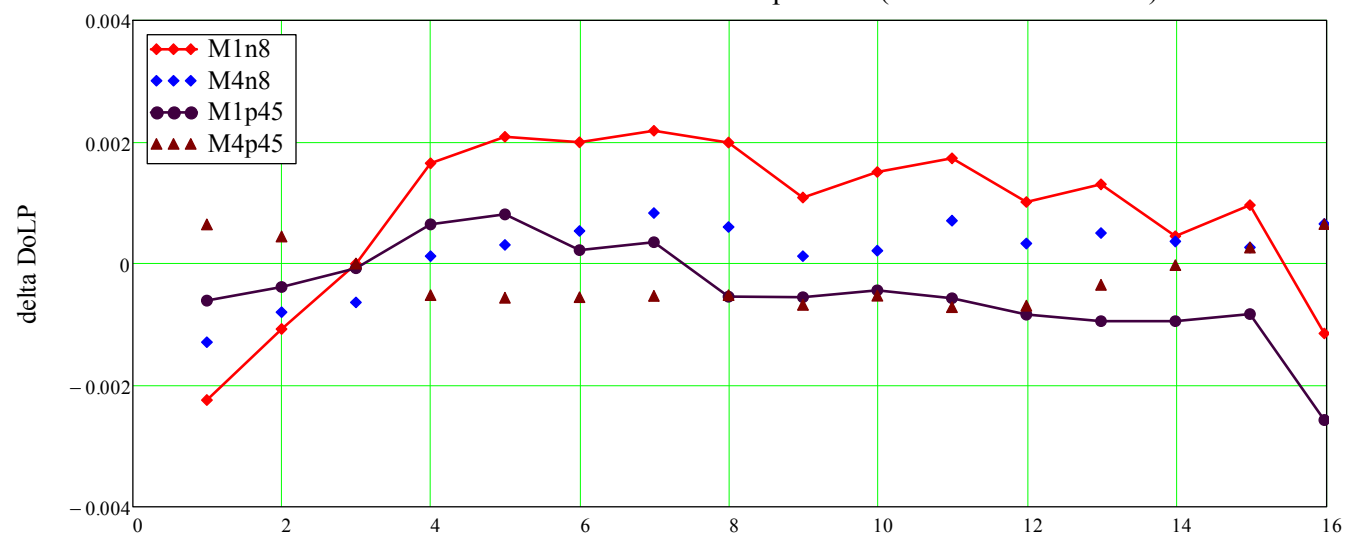

Fig.9: Band M1 and M4 detector number

A summary of T-SIRCUS measurement result is the following:

1. T-SIRCUS data illustrates enhanced polarization at band edges similar to the Raytheon polarization model.

2. A factor in the poor model correlation for M4 may be inadequate component measurements 
3. VIIRS polarization performance characterization has a dependency on source spectral distribution ranging between 0.0035 and 0.0010 .

4. Based upon the analysis T-SIRCUS polarization after correction for SIS100-2 spectral effects agree with FP-11 results within $+/-0.002$, see fig.9.

\section{T-SIRCUS MEASUREMENT RESULTS ${ }^{19}$}

Based on T-SIRCUS test data, a fourth order Fourier series was used to fit the sensor response over polarization angle. The Fourier components were used to determine the DoLP and phase angle. This was done separately for each detector, wavelength, and scan angle. The Fourier components were weighted by the spectral transmittance of the system, and then integrated over the bandpass to determine the band dependent DoLP and phase angle for comparison to FP-11 / FP11 ' polarization measurements.

The measured dn (offset corrected detector response) for each polarizer angle at $408 \mathrm{~nm}$ is shown in Fig. 9(a) for all detectors (at +45 degrees scan angle, HAM side 1); the symbols represent the measured $d n$ and the solid lines denote the calculated Fourier series. Each detector is represented by a different symbol / color as defined in the legend. Similarly, the measurements and fits for $421 \mathrm{~nm}, 546 \mathrm{~nm}$, and $559 \mathrm{~nm}$ (at +45 degrees scan angle, HAM side 1) are shown by the symbols and solid lines in Fig. 9 (b, c, and d). Note that the lines fit the data very well and closely follow a two-cycle oscillation. This indicates that the second order Fourier components dominate. Note that this is considerable variation for some wavelengths in terms of both amplitude and phase angle. The cases shown in Fig. 9 are indicative of all the TSIRCUS measurements.
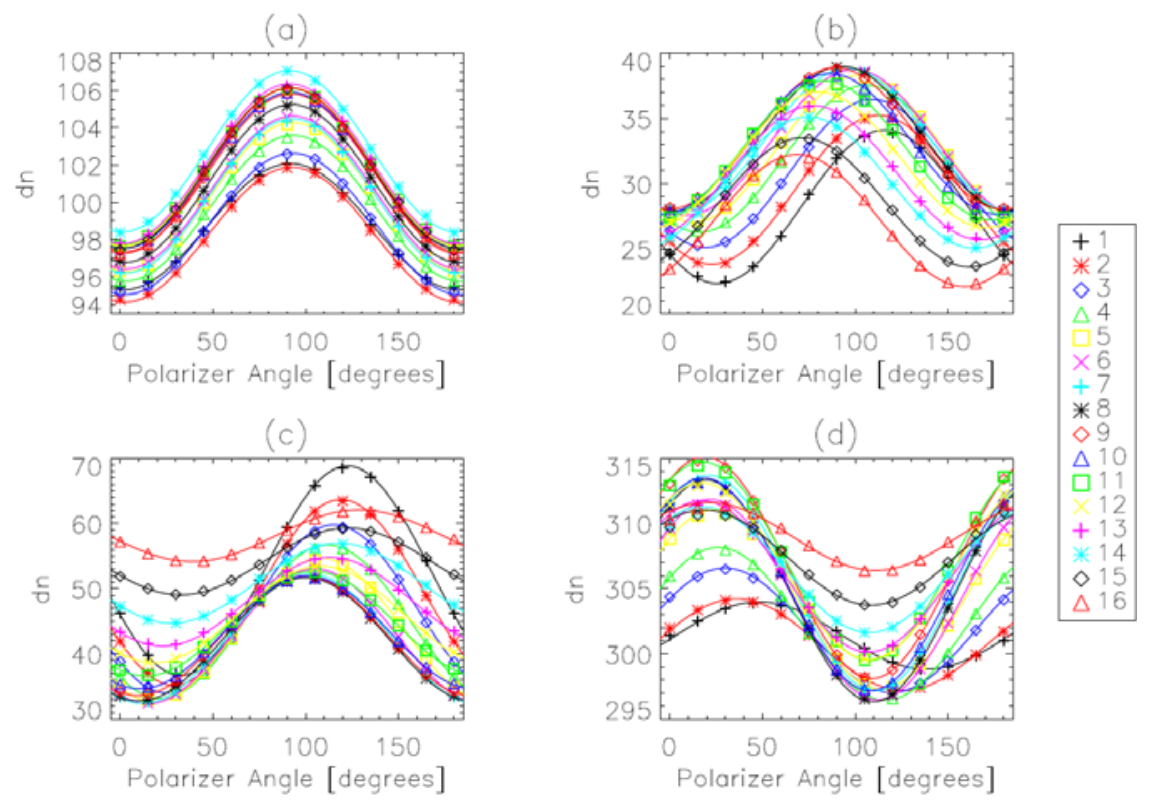

Figure 9. Measured dn versus polarizer angle [degrees] for M1 at $408 \mathrm{~nm}$ (a), M1 at $421 \mathrm{~nm}$ (b), M4 at $546 \mathrm{~nm}$ (c), and M4 at $559 \mathrm{~nm}$ (d) at +45 degrees scan angle, HAM side 1 . The legend defines the different symbol / color combinations which correspond to each detector.

The spectrally weighted DoLP for M1 (+45 degrees scan angle, HAM side 1, resampled to $1 \mathrm{~nm}$ ) as a function of wavelength is shown in Fig. 10(a) indicates that the DoLP increases on the sides of the bandpass, and then decreases in the middle. A similar plot for M4 ( +45 degrees scan angle, HAM side 1, resampled to $1 \mathrm{~nm}$ ) is shown in Fig. 10(b). For the M4 case, the DoLP oscillates in the center of the bandpass; the minimums in DoLP correspond to phase angle shifts. The cases not shown (M1, -8 degrees scan angle, HAM side 1; M4, -8 degrees scan angle, HAM side 0; and M4, -8 degrees scan angle, HAM side 1) show similar results. The corresponding ray trace model results are shown in Fig. 10(c) and 10(d) for M1 and M4, respectively. Note that the model and measurements agree in the general shape and characteristics of the DoLP versus wavelength. For M1, the modeled bandpass is slightly wider than the measurement; for M4, the modeled bandpass is also wider and the phase angle shifts that occur in the center of the bandpass were not captured by the model. 
(a)

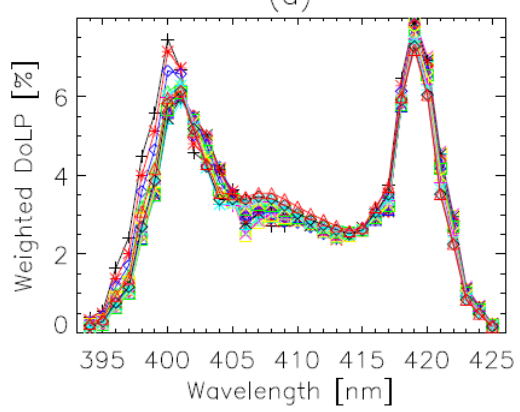

(c)

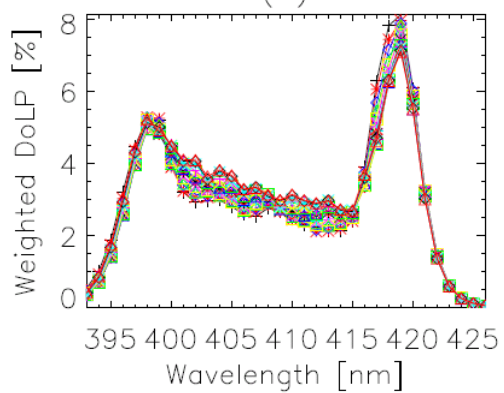

(b)

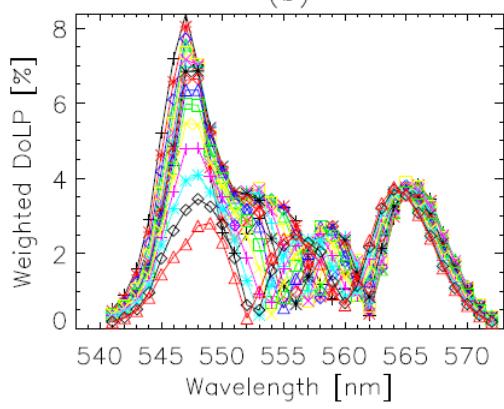

(d)

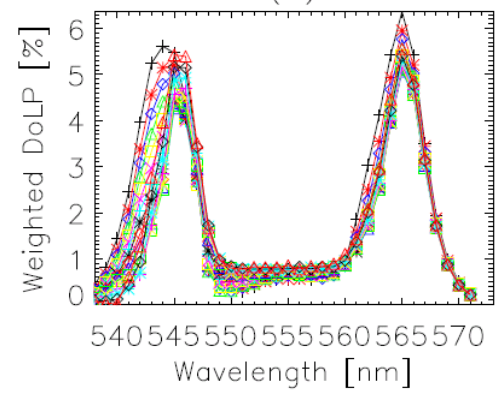

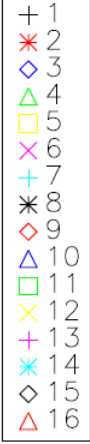

Figure 10. Measured, spectrally weighted DoLP versus wavelength for M1 (a) and M4 (b); modeled weighted DoLP versus wavelength for M1 (c) and M4 (d). All plots show data at +45 degrees scan angle, HAM side 1 . The legend defines the different symbol / color combinations which correspond to each detector.

(a)

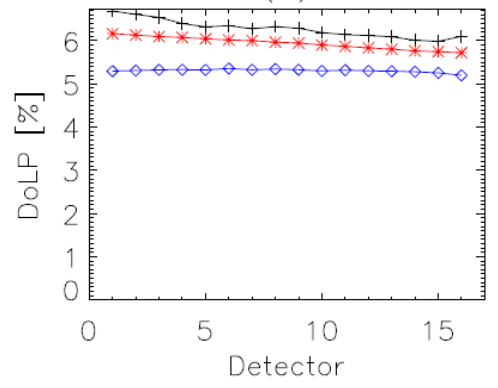

(c)

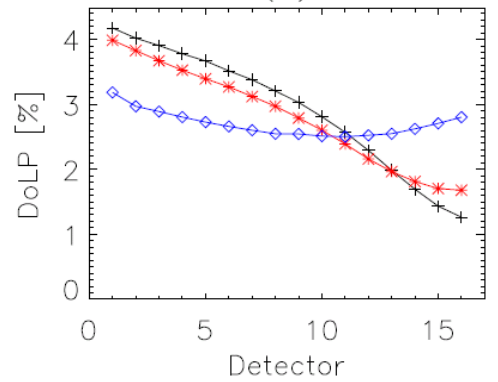

(b)

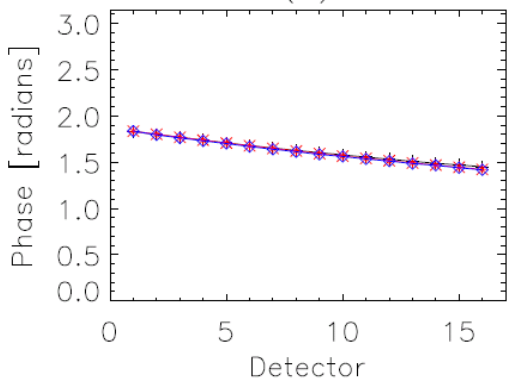

(d)

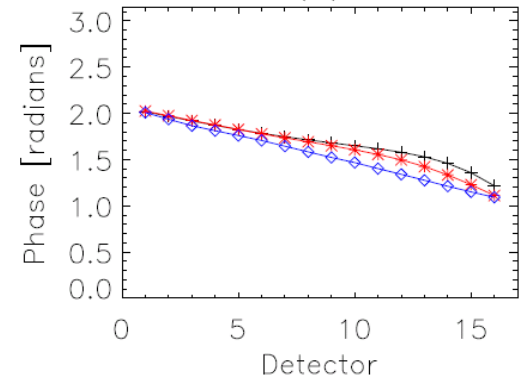

Figure 10. Plots of the DoLP and phase showing: M1 at +45 degrees scan angle, HAM side 1 in (a) and (b) as well as M4 at +45 degrees scan angle, HAM side 1 in (c) and (d). Black '+'s indicate T-SIRCUS measurements, red '*'s denote broadband measurements, and blue ' $\diamond$ 's refer to the model.

The band dependent DoLP and phase angles are shown for +45 degrees scan angle, HAM side 1 in Figure 10 (all other cases show similar results). In general, the T-SIRCUS results agree reasonably well with the broadband measurements, 
with differences of up to $\sim 0.5 \%$. Note that the M1 and M4 bandpasses were not critically sampled (the laser bandpass was less than $1 \mathrm{~nm}$ ). Model differences with measurements are small for M1, but larger for M4; for M4, the detector dependence was not captured well by the model. The phase angles are very similar for the M1 cases; however, for M4, the phase angle differences between T-SIRCUS and broadband measurements were larger for higher number detectors, up to $\sim 14$ degrees.

\section{CONCLUSION}

The polarization sensitivity measurement of the VIIRS/J1 instruments, FP11, was performed. While polarization characterization uncertainty was less than $0.3 \%$, the maximum polarization sensitivity for M1, m2, M3, and M4 bands was measured to be $6.4 \%, 4.4 \%, 3.1 \%$, and $4.3 \%$, respectively, exceeding the specification. The polarization sensitivity measurement of VIIRS/J1 AOA has similar magnitude of polarization sensitivity for M1 to M4 bands.. The FRED polarization ray trace model indicated the increased polarization sensitivity for M1 to M4 bands was mainly due to the S and $\mathrm{P}$ polarization split at the leading edge and the trailing edge of newly manufactured focal plane spectral bandpass filter in front of the VISNIR detectors. The polarization sensitivity of M1 and M4 bands were measured on the edges and peak of the bandpass filter using a monochromatic light from the T-SIRCUS which confirmed the FRED model's predictions. To improve the confidence in the polarization scan angle interpolation, VIIRS/J1 polarization measurement were performed at additional scan angles, FP-11', using a white illumination light source. The repeatability of polarization sensitivity at $-8^{\circ}$ scan angle between FP-11 and FP-11' was consistent with polarization measurement uncertainty. In general, polarization sensitivity vs. scan angle interpolation uncertainty was determined to be less than $+/-$ 0.001. The good agreement between the FRED model, FP-11, FP-11' and the T-SIRCUS measurements increases the confidence in the FRED model and its use to predict future on-orbit polarization performance and its ability (with suitable coating file updates) to predict $\mathrm{J} 2$ performance.

\section{ACKNOWLEDGEMENTS}

The authors wish to thank the VIIRS operations crew, who control the workings of VIIRS during the polarization tests for their patience and willingness to explain what was going on during the tests. Thanks also go to Jolene Vallejo for her administrative help. Also thanks go to JPSS management for their support of this work.

\section{APPENDIX}

Table A1. Translation between VIIRS and Mueller Matrix terminology

\begin{tabular}{|c|c|c|}
\hline VIIRS Terminology & Mueller Matrix Terminology & Comments \\
\hline $\begin{array}{c}\text { Polarization sensitivity or Degree of } \\
\text { Linear Polarization (DoLP) }\end{array}$ & Linear Diattenuation & DoLP=sqrt(A2 $\left.2^{\wedge} 2+\mathrm{B} 2 \wedge 2\right)$ \\
\hline A2 Fourier Coefficient & Mueller matrix element $\mathrm{m}_{12}$ & \\
\hline B2 Fourier Coefficient & Mueller matrix element $\mathrm{m}_{13}$ & \\
\hline Phase Angle & Angle of Linear Diattenuation & Phase Angle $=0.5^{*} \tan ^{-1}(\mathrm{~B} 2 / \mathrm{A} 2)$ \\
\hline
\end{tabular}

\section{REFERENCES}

${ }^{1}$ Carl Schueler, J. Ed Clement, Russ Ravella, Jeffery J. Puschell, Lane Darnton, Frank DeLuccia, Captain Tanya Scalione, Hal Bloom, Hilmer Swenson, "VIIRS Sensor Performance", Geoscience and Remote Sensing Symposium, 2003. IGARSS '03. Proceedings. 2003 IEEE International

Year: 2003, Volume: 1, Pages: 369 - 372 vol.1

${ }^{2}$ D. Goldstein, Polarized Light, 2nd. Ed., Chapter 9, Marcel-Dekker (2003)

${ }^{3}$ Jim Young, “J1 AOA Polarization Comments.pptx”, unpublished, (2012) 
${ }^{4}$ E.Waluschka, "VIIRS polarization testing", Proc. SPIE 7452, Earth Observing Systems XIV, 745209 (August 21, 2009

${ }^{5}$ Bolder Vision Optik, 4730 Walnut Street, Suite \#102, Boulder, Colorado, 80301

${ }^{6}$ MOXTEK, Inc., 452 W 1260 N, Orem, UT 84057

${ }^{7}$ Sonoma C165-210-JB NIR Blocking Filter, Sonoma Photonic Incorporated, 1750 Northpoint Parkway, Santa Rosa, CA

${ }^{8}$ Raytheon, Performance Specification, Sensor Specification for the VIIRS, PS154640-101 Rev. D (2008)

${ }^{9}$ G. Meister, E. J. Kwiatkowska, B. A. Franz, F. S. Patt, G. C. Feldman, and C. R. McClain, "Moderate-Resolution Imaging Spectroradiometer ocean color polarization correction," Appl. Opt. 44, 5524 - 5535 (2005).

${ }^{10}$ H. R. Gordon, T. Du, and T. Zhang, "Atmospheric correction of ocean color sensors: analysis of the effects of residual instrument polarization sensitivity,” Appl. Opt. 36, 6938 - 6948 (1997).

${ }^{11}$ E. Waluschka, "MODIS polarization measurements and simulation and the $4 \theta$ effect", Proc. SPIE 3121, Polarization: Measurement, Analysis, and Remote Sensing, 278 (October 3, 1997)

${ }^{12}$ Moyer, D., McIntire, J., Waluschka, E., Xiong, X., and DeLuccia, F., "JPSS-1 VIIRS pre-launch polarization testing and performance," IEEE T. Geosci. Remote (2015). In press.

${ }^{13}$ E. Fest, "VIIRS polarization sensitivity testing and analysis", Proc. SPIE 7461, 746102 (2009)

${ }^{14}$ R. Miller and G. Hennessey, "Infrared coatings for 2-15 micron”, Proc. SPIE 0050, 119 (1974)

${ }^{15}$ Moeller, C., Schwarting, T., and Moyer, D., “JPSS J1 T-SIRCUS RSR,” Unpublished (2015)

${ }^{16}$ Kevin $<$ include ocean color analysis reference here $>$

${ }^{17}$ S. W. Brown, G. P. Eppeldauer, and K. R. Lykke "Facility for Spectral Irradiance and Radiance Calibrations using Uniform Sources,” Appl. Op. 45, 8218-8237 (2006).

${ }^{18}$ Barnes, R. A., Brown, S. W., Lykke, K. R., Guenther, B., Xiong, X., and Butler, J. J., "Comparison of two methodologies for calibrating satellite instruments in the visible and near infrared," Proc. of SPIE 7862

${ }^{19}$ McIntire, J., Young, J. B., Moyer, D.,Waluschka, E., Oudrari, H., and Xiong, X., “Analysis of JPSS J1 VIIRS polarization sensitivity using the NIST T-SIRCUS," Proc. of SPIE 9607 (2015). In press. 